\title{
Angioleiomioma en cavidad oral uuna neoplasia rara en un sitio poco común». Reporte de un caso y revisión de la literatura.
}

\author{
Angioleiomyoma in the oral cavity "a rare neoplasm in an \\ unusual site».Case report and literature review. \\ María Fernanda Partida González,* Gustavo Pedro Díaz Colmenares,* Anabell Alvarado Sánchez,* \\ Yamely Ruiz Vázquez, ${ }^{\ddagger}$ Daniel Alejandro Guerra Leal ${ }^{\S}$
}

\section{RESUMEN}

El angioleiomioma (AL) es una neoplasia benigna, bien circunscrita y de crecimiento lento, que representa 5\% de las neoplasias de tejidos blandos y cuya etiología es desconocida. Se origina del músculo liso, mayormente de las paredes de los vasos sanguíneos; su localización es más frecuente en extremidades, siendo raros en la región de cabeza y cuello, y más aún en cavidad bucal. Histológicamente la lesión se caracteriza por ser un nódulo bien encapsulado con proliferación de fascículos de músculo liso maduro alrededor de la luz de los vasos sanguíneos, cuyas células suelen ser positivas a marcadores de inmunohistoquímica como alfa actina de músculo liso, desmina, HHF35, miosina, calponina y H-caldesmon. El tratamiento actual es la escisión quirúrgica completa con una tasa de recurrencia prácticamente nula. El objetivo es resaltar la importancia del diagnóstico y el manejo correcto de las lesiones intraorales a través de la presentación de un caso clínico de un leiomioma vascular localizado en región nasolabial, además de hacer la revisión de la literatura correspondiente.

Palabras clave: Angioleiomioma, leiomioma vascular, angiomioma, músculo liso, leiomioma oral, leiomioma bucal.

\section{ABSTRACT}

The angioleiomyoma $(\mathrm{AL})$ is a benign neoplasm, well circumscribed and slow growing, that represents $5 \%$ of the soft tissue neoplasms, whose etiology is unknown. It originates from smooth muscle, mostly from the walls of blood vessels; regarding its location, it more frequently appears in the extremities, being rare in the head and neck region, and even more so in the oral cavity. Histologically, the lesion is characterized by being a well encapsulated nodule with proliferation of mature smooth muscle bundles around the lumen of the blood vessels, whose cells are usually positive for immunohistochemical markers such as alpha smooth muscle actin, desmin, HHF35, myosin, calponin and H-caldesmon. The current treatment is complete surgical excision having zero recurrence rate. The objective of the following article is to educate on the importance of correct diagnosis and management of intraoral lesions through the presentation of a clinical case of a vascular leiomyoma located in the nasolabial region, in addition to reviewing the corresponding literature.

Keywords: Angioleiomyoma, vascular leiomyoma, angiomyoma, smooth muscle, oral leiomyoma, buccal leiomyoma.

\section{INTRODUCCIÓN}

$\mathrm{E}$ I angioleiomioma (AL) es una neoplasia benigna, bien circunscrita y de crecimiento lento, descrita por Virchow en 1854 como tuberculum dolorosum, y posteriormente Stout en 1937 le acuñó el término «leiomioma vascular». ${ }^{1,2}$ Se considera un subtipo solitario de leiomioma, ${ }^{3}$ compuesto por músculo liso y numerosos

\footnotetext{
* Médico Cirujano Odontólogo, pasante del Tecnológico de Monterrey.

₹ Maestra en Patología y Medicina Bucal, Universidad Nacional Autónoma de México.

$\S$ Cirujano Maxilofacial, Coordinador del Centro Académico de Atención Odontológica (CAAD) del Tecnológico de Monterrey, Escuela de

Medicina y Ciencias de la Salud.

Instituto Tecnológico y de Estudios Superiores de Monterrey, Escuela de Medicina y Ciencias de la Salud. México.

Recibido: 06 de diciembre de 2021. Aceptado: 11 de enero de 2022.

Citar como: Partida GMF, Díaz CGP, Alvarado SA, Ruiz VY, Guerra LDA. Angioleiomioma en cavidad oral «una neoplasia rara en un sitio poco común». Reporte de un caso y revisión de la literatura. Rev ADM. 2022; 79 (1): 48-53. https://dx.doi.org/10.35366/103818
}

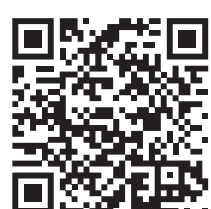


vasos sanguíneos de pared delgada. En la actualidad, para muchos autores, esta lesión se considera como parte del espectro perivascular junto con el miopericitoma y el miofibroma; sin embargo, para otros autores, las características morfológicas del AL pueden, hasta cierto punto, sobreponerse a las del miopericitoma, haciendo que la diferencia entre estas dos lesiones pueda ser subjetiva en muchas ocasiones. ${ }^{4,5}$ La etiología es desconocida, pero se han descrito posibles factores etiológicos como el trauma menor, desbalance hormonal, estasis venosa, disminución de la irrigación venosa y alteraciones genéticas.., $6-8$

El AL representa 5\% de las neoplasias de tejidos blandos y puede llegar a representar hasta $50 \%$ de todos los leiomiomas superficiales; sin embargo, en cavidad oral la incidencia es de 0.06 a $0.065 \%$ y esto se relaciona con la escasa presencia de músculo liso en esta área. ${ }^{4,9}$ Los sitios de predilección son labios, paladar duro, carrillos, mucosa yugal, encía y lengua. ${ }^{10,11}$

Contrario a lo observado en el leiomioma convencional, donde la prevalencia es mayor en mujeres y en sitios como extremidades inferiores y tronco, el AL es más común en hombres entre la cuarta y sexta década de la vida, específicamente en las áreas de cabeza y cuello. ${ }^{4-6,10,12}$

Clínicamente se ha descrito como una masa asintomática, sésil, solitaria, móvil, esférica y bien circunscrita. Su coloración es muy similar a la de la mucosa adyacente, aunque en ocasiones se observa con parches rojos. Su tamaño promedio varía desde 1 hasta $3 \mathrm{~cm}$ de diámetro.,5,13-16

Respecto a la imagenología, la resonancia magnética nuclear (RMN) y la tomografía computarizada (TC) con medio de contraste son los estudios con mayor utilidad para la ubicación de la lesión y planificación de la cirugía. 6,12

Microscópicamente se caracteriza por la proliferación de fascículos de músculo liso maduro alrededor de la luz de los vasos sanguíneos, encapsulada por una capa de tejido conectivo colagenizado. Las células musculares lisas suelen ser positivas a marcadores de inmunohistoquímica como alfa actina de músculo liso, desmina, HHF35, miosina, calponina y $\mathrm{H}$-caldesmon.

En la actualidad, el tratamiento consiste en la escisión quirúrgica y en ocasiones acompañado del ligado o cauterización de los vasos sanguíneos adyacentes. ${ }^{7,12}$

\section{PRESENTACIÓN DEL CASO}

Se presenta el caso de un paciente femenino, de 23 años de edad, quien acudió al Centro Académico de Atención Odontológica (CAAD) del Tecnológico de Monterrey por presentar aumento de volumen en la región del ala nasal izquierda. La paciente refiere antecedentes de polineuro- patía desmielinizante inflamatoria crónica (CIDP, por sus siglas en inglés) y estar bajo tratamiento con inmunoglobulinas. Refiere presentar la lesión desde la infancia, pero que en los últimos tres meses ha aumentado de tamaño con un curso indolente.

De acuerdo con lo referido por la paciente y basado en la literatura, los pasos de examinación clínica indicados son observación, palpación, valoración de la movilidad, medición de la lesión, toma de radiografía y fotografía. Además, se debe registrar el sitio en que se encuentra, forma, morfología, color, bordes, consistencia, tamaño y descripción exacta de la lesión que se muestre en la fotografía tomada. ${ }^{17}$

Con base en el protocolo de atención de lesiones neoplásicas, en la exploración clínica se encontró un nódulo de aproximadamente $1 \mathrm{~cm}$ de diámetro, móvil, firme a la palpación, de color similar a la piel adyacente con áreas ligeramente violáceas y que dentro de la mucosa bucal ocasiona pérdida del surco mucogingival (Figura 1A). La radiografía periapical no mostró datos o hallazgos patológicos en órganos dentarios adyacentes o tejido óseo (Figura 1B).

Se realizó punción aspirativa previo a la incisión sin obtener salida de ningún contenido, esto con la finalidad de descartar una lesión de origen vascular y como parte del protocolo de biopsias en lesiones intraorales con coloración diferente a la mucosa sana, para evitar hemorragia masiva y/o persistente. ${ }^{17}$

Debido al tamaño y localización de la lesión se decidió realizar biopsia escisional, indicada en lesiones menores o hasta de $2.5 \mathrm{~cm}$ de diámetro, bajo anestesia local infiltrativa con lidocaína al 2\% con epinefrina 1:100,000.

Se realizó una incisión semilunar modificada desde el órgano dentario 1.1 hasta el 2.3. Posteriormente, se realizó disección roma con pinzas de mosco curvas para localizar la lesión, disecarla y extirparla por completo (Figura 1C). Por último, se lavó el lecho quirúrgico con solución fisiológica y peróxido de hidrógeno, se corroboró hemostasia y se colocaron puntos aislados de sutura (Figura 1D). La muestra obtenida midió $1.2 \mathrm{~cm}$ en su diámetro mayor y fue fijada en formol al $10 \%$ y enviada para su estudio histopatológico, el cual es de suma importancia para normar el tratamiento a seguir de la lesión. ${ }^{18}$

El análisis histopatológico de la lesión mostró una neoplasia a expensas de la proliferación de células fusiformes de citoplasma eosinófilo con núcleos ovales, alargados y bordes romos; con un arreglo en fascículos cortos que rodeaban a vasos sanguíneos ectásicos llenos de eritrocitos, además de hemorragia reciente y algunos depósitos de hemosiderina. La lesión se encontraba rodeada por 
una delgada cápsula de tejido conectivo fibroso denso bien vascularizado (Figura 2A-D). Se realizó anticuerpo de inmunohistoquímica para $\mathrm{H}$-caldesmon, con resultado intenso positivo citoplasmático, confirmando así el diagnóstico de leiomioma vascular (Figura 2E y F).

\section{DISCUSIÓN}

El angioleiomioma es una neoplasia benigna de crecimiento lento y bien circunscrita. ${ }^{12}$ Se conoce también como angiomioma, leiomioma vascular o angiomioma dérmico y es un subtipo histológico del leiomioma. 7,19,20 La Organización Mundial de la Salud (OMS) lo describe como un tumor de origen perivascular, pero con etiología desconocida. ${ }^{4}$ Se han postulado tres hipótesis de su origen: 1) de células indiferenciadas mesenquimatosas anormales, 2) del músculo liso de las paredes de las venas y músculo erector de pelo o 3) ambas hipótesis juntas. ${ }^{12,21,22}$ Asimismo, se cree que factores como el trauma menor, estasis venosa, disminución de la irrigación venosa, desbalance hormonal (estrógenos) y alteraciones genéticas (genes BRAF, NF1, NOTCH2 y NOTCH3) intervienen en su origen. $., 7,8,23$

El AL se ha reportado en un rango amplio de edades, aunque es más común en pacientes que están entre la cuarta y sexta década de la vida. ${ }^{6,24}$ Cuando aparece en pacientes infantiles se le llama épulis (leiomiomatoso) congénito. ${ }^{19}$ Cuando está presente en extremidades o tronco, es más común en mujeres (proporción 3:2 mujer/ hombre), mientras que en cabeza y cuello es más común en hombres (proporción 1:3 mujer/hombre). 4,6,7,10

Sólo de 0.06 a $0.065 \%$ de los AL se encuentran en cabeza y cuello y de éstos de 64 a $66.2 \%$ se encuentran en la cavidad oral. 4,9,19,25 Los sitios con mayor frecuencia
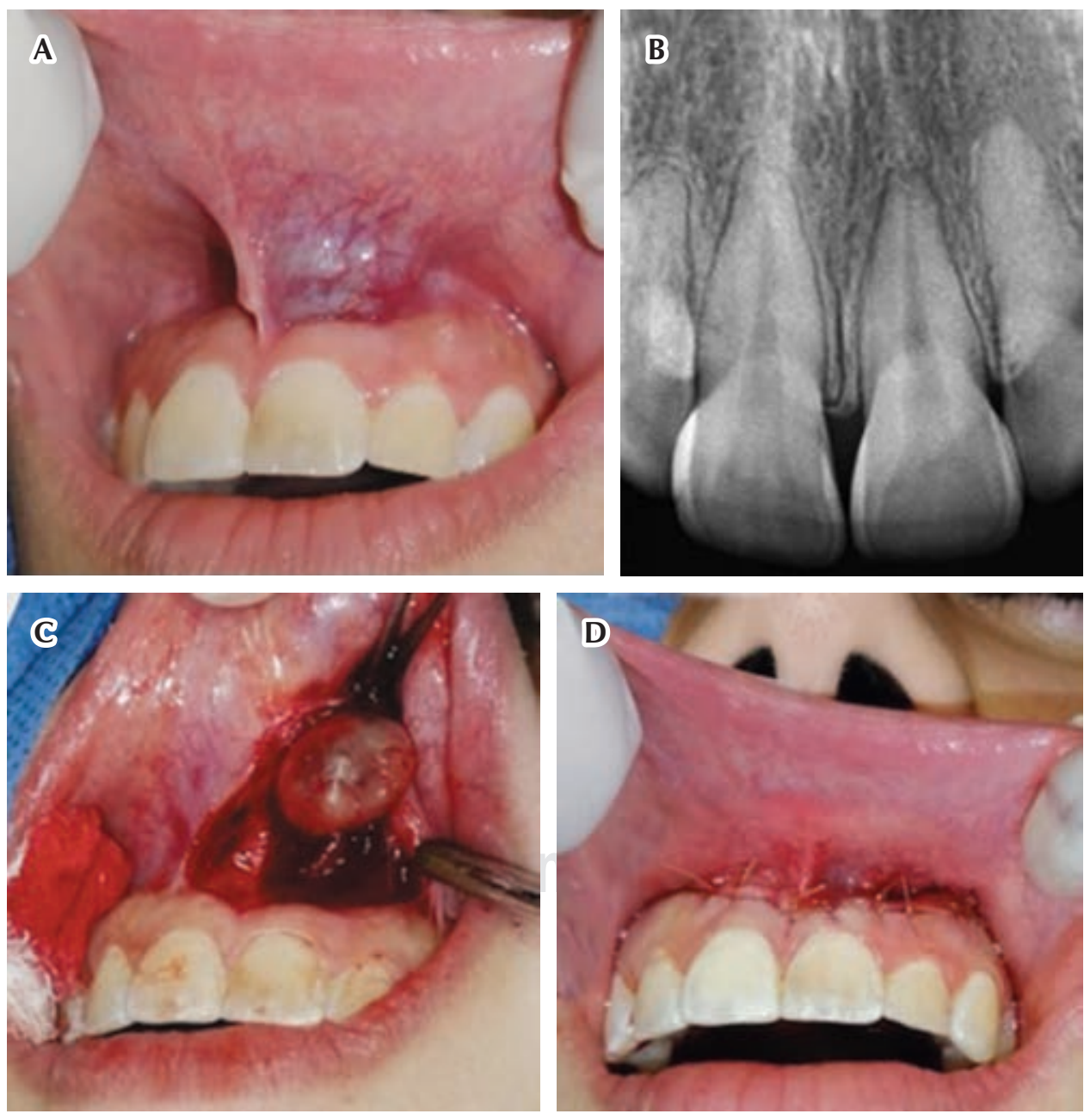

Figura 1:

A) Lesión intraoral. B)

Radiografía periapical de la lesión. C) Extirpación de la lesión. D) Puntos de sutura simples. 

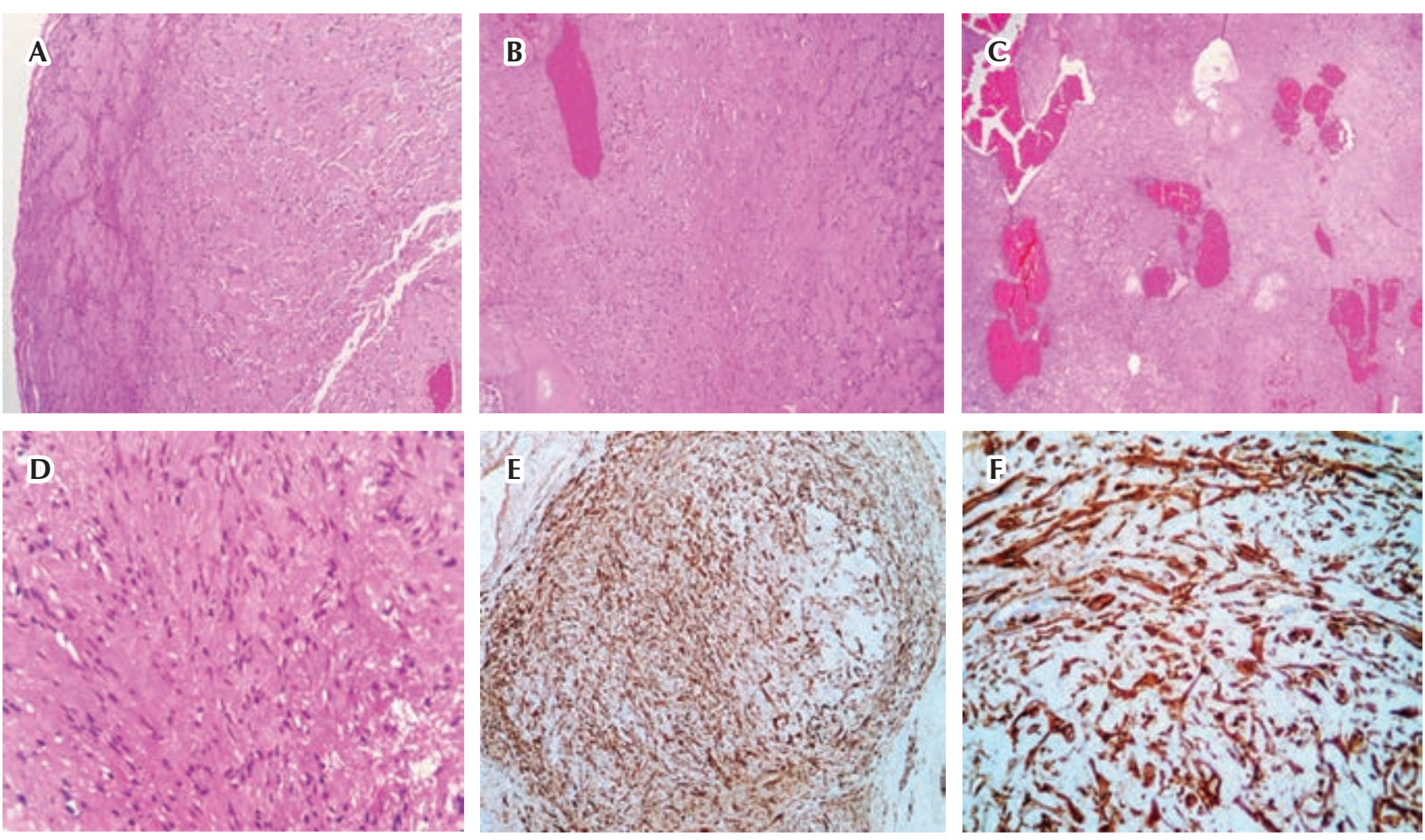

Figura 2: A-C) H\&E 10x. Fascículos de células musculares lisas alrededor de vasos sanguíneos ectásicos. D) H\&E 40×. Células fusiformes de citoplasma eosinófilo con núcleos basófilos de bordes romos. E-F) Inmunohistoquímica para H-caldesmon 10× y 40×, con marcaje intenso positivo citoplasmático en las células neoplásicas.

de aparición son: labios (48.6\%), paladar (21.1\%), mucosa bucal $(9.2 \%)$, lengua $(9.2 \%)$, mandíbula $(8.3 \%)$, fondo vestibular superior $(0.9 \%)$, fondo vestibular inferior $(0.9 \%)$, piso de boca $(0.9 \%)$ y encía $(0.9 \%) .6,8-10,25$

En 1973 Morimoto estudió 241 casos de AL y los clasificó en tres tipos histológicos:

1. Sólido: es el más común. Se ve músculo liso y pequeños canales vasculares. Es tres veces más frecuente en mujeres que hombres $(3: 1)$.

2. Venoso: se distingue fácilmente por paredes musculares gruesas.

3. Cavernoso: es el menos común. Se ven canales vasculares dilatados con menos músculo liso. Es cuatro veces más usual en hombres $(4: 1)$.

Y los asoció en dos grupos:

1. Grupo mayor: tumores de extremidades, más común el tipo sólido y con dolor. Proporción 2:1 mujer/hombre.
2. Grupo menor: tumores en cabeza, más común el tipo venoso y son asintomáticos. Más comunes en hombres.

De estos tipos, el venoso fue el más asociado a la cavidad oral. $4,7,22,26$

Los estudios con imagenología de tipo resonancia magnética (RM) muestran una imagen homogénea e isointensa al músculo esquelético. ${ }^{7,9}$ Diversos autores mencionan que es el estudio más útil para la planificación de la cirugía, ya que permite ver si hay una involucración con estructuras nerviosas. ${ }^{6,12}$

A pesar de que las características clínicas y de imagen nos van acercando al diagnóstico de $A L$, su baja incidencia en cavidad oral y su similitud con otras lesiones nos obliga a descartar patologías a través del análisis histopatológico, ${ }^{24}$ tales como: lipoma, fibroma, neurofibroma, neurilemoma, mucocele, adenoma pleomorfo, linfangioma, hemangioma, quiste dermoide, schwannoma, tumor mesenquimatoso, quiste de inclusión, angiofibroma, miofibroma, leiomiosarcoma, 
miopericitoma, angiolipoma, quiste ganglionar y neuroma traumático. ${ }^{4,7-10,19,25}$

Es por ello que ni las características clínicas o de imagen pueden dar un diagnóstico definitivo, ya que podrían llevar a una identificación incorrecta de la patología. El único método que puede definir el diagnóstico es el análisis histológico a través de tinciones y/o marcadores inmunohistoquímicos. ${ }^{9,19,21}$ Las tinciones como tricrómico de Masson, hematoxilina-eosina, azul alcián, van Gieson y ácido peryódico de Schiff (PAS, por sus siglas en inglés) son útiles para el diagnóstico. ${ }^{9,12}$ Asimismo, hay una gran cantidad de marcadores inmunohistoquímicos de gran utilidad, algunos más específicos que otros, siendo positivo para actina del músculo liso (SMA, por sus siglas en inglés), desmina, $\mathrm{H}$-caldesmon, vimentina, actina HHF35 y negativo para S-100, CD-68, CD-34, citoqueratina, antígeno de membrana epitelial (EMA, por sus siglas en inglés). $4,6,8,10,19$

El tratamiento se mantiene a la escisión quirúrgica con márgenes de 1-2 mm y en ocasiones se complementa con el ligado del vaso alimentante. Durante la escisión se debe tener cuidado cuando la neoplasia esté en contacto con haces neuromusculares. La transformación maligna o metástasis son sumamente raras y una vez retirada la lesión su recidiva es prácticamente nula.

\section{CONCLUSIONES}

La atención integral al paciente en el área odontológica tiene como objetivo brindar un tratamiento adecuado, abordando la problemática desde el diagnóstico, la elección de tratamiento y resolución de ésta de la manera correcta, siempre basado en la literatura y adaptado al paciente según su estado de salud sistémico.

Las competencias adquiridas en la práctica clínica del alumno dan como resultado el manejo adecuado de los diferentes tipos de neoplasias, identificando la lesión, definiendo el tipo de biopsia necesaria, así como el abordaje quirúrgico ideal y pronóstico de la misma.

En este caso se aplicaron las competencias adquiridas en el manejo del $\mathrm{AL}$, la cual es una neoplasia infrecuente en cabeza y cuello, y más en la cavidad oral; el caso presentado muestra un paciente con un $\mathrm{AL}$ en fondo de saco y en la región del ala nasal izquierda, siendo éste un sitio que, de acuerdo con la literatura, no es el más frecuente o habitual. La lesión cumplía con todas las características clínicas y de análisis inmunohistoquímicos. Como parte del desarrollo profesional del alumno, se llegó a la conclusión, con respecto a la lesión, de que se debe hacer más investigación genética para aclarar su etiología y es de suma importancia la imagenología para conocer la extensión y así poder realizar una cirugía óptima. De igual manera, se reitera que el análisis histológico es la única manera para poder establecer el diagnóstico definitivo de la lesión.

\section{REFERENCIAS}

1. Virchow R. Ueber makroglossie und pathologische neubildung quergestreifter muskelfasern. Virchows Arch (Pathol Anat). 1854; 7: 126-138.

2. Stout AP. Solitary cutaneous and subcutaneous leiomyoma. Am J Cancer. 1937; 29: 435-469.

3. Peric B, Vidakovic B, Grgic N, Manojlovic S, Cavka H. Angiomyoma of the upper lip - case report and review of the literature. Acta Clin Croat. 2019; 58 (1): 183-186. doi: 10.20471/acc.2019.58.01.24.

4. Thakker T, Anehosur V, US D, Anand J, Kumar N. Gingival angioleiomyoma - A rare case report. Oral and Maxillofacial Surgery Cases. 2019; 5 (3): 100114. doi: 10.1016/j.omsc.2019.100114.

5. Goldblum JR, Folpe AL, Weiss SW. Enzinger and Weiss's soft tissue tumors. 7th ed. Philadelphia, PA, USA: Elsevier; 2019.

6. da Silva LAB, de Medeiros AMC, de Oliveira PT, da Silveira EJD, Miguel MCDC. Angioleiomioma oral: relato de um caso e revisão dos achados atuais. J Vasc Bras. 2017; 16 (1): 68-72.

7. Ramesh P, Annapureddy SR, Khan F, Sutaria PD. Angioleiomyoma: a clinical, pathological and radiological review. Int J Clin Pract. 2004; 58 (6): 587-591. doi: 10.1111/j.1368-5031.2004.00085.x.

8. Ishikawa S, Fuyama S, Kobayashi T, Taira Y, Sugano A, lino M. Angioleiomyoma of the tongue: a case report and review of the literature. Odontology. 2016; 104 (1): 119-122. doi: 10.1007/ s10266-014-0175-0.

9. Tsuji T, Satoh K, Nakano H, Kogo M. Clinical characteristics of angioleiomyoma of the hard palate: report of a case and an analysis of the reported cases. J Oral Maxillofac Surg. 2014; 72 (5): 920-926. doi: 10.1016/j.joms.2013.11.008.

10. Giudice A, Bennardo F, Buffone C, Brancaccio Y, Plutino FM, Fortunato L. Clinical and immunohistochemical features of oral angioleiomyoma: a comprehensive review of the literature and report of a case in a young patient. Case Rep Dent. 2019; 2019: 2498353. doi: 10.1155/2019/2498353.

11. Gueiros LA, Romañach MJ, Pires-Soubhia AM, Pires FR, Paes-deAlmeida O, Vargas PA. Angioleiomyoma affecting the lips: report of 3 cases and review of the literature. Med Oral Patol Oral Cir Bucal. 2011; 16 (4): e482-e487.

12. Sayit E, Sayit AT, Zan E, Bakirtas M, Akpinar H, Gunbey HP. Vascular leiomyoma of an extremity: Report of two cases with MRI and histopathologic correlation. J Clin Orthop Trauma. 2014; 5 (2): 110-114. doi: 10.1016/j.jcot.2014.04.001.

13. Bajpai M, Pardhe N, Kumar M. Angioleiomyoma of gingiva masquerading as pyogenic granuloma. J Coll Physicians Surg Pak. 2016; 26 (7): 631-632.

14. Inaba $\mathrm{T}$, Adachi $\mathrm{M}$, Yagisita $\mathrm{H}$. A case of angioleiomyoma in the buccal space. Odontology. 2015; 103 (1): 109-111. doi: 10.1007/ s10266-013-0128-z.

15. Kim HY, Jung SN, Kwon H, Sohn WI, Moon SH. Angiomyoma in the buccal space. J Craniofac Surg. 2010; 21 (5): 1634-1635. doi: 10.1097/SCS.0b013e3181ef67f1.

16. Ribeiro JL, Costa FH, Cavalcante AS, Kaminagakura E, Carvalho YR, Anbinder AL. Vascular leiomyoma in the oral cavity - Report of two cases. J Clin Exp Dent. 2019; 11 (6): e582-e586. doi: 10.4317/ jced.55684. 
17. Chan MH, Wolf JC. Biopsy techniques and diagnoses \& treatment of mucocutaneous lesions. Dent Clin North Am. 2012; 56 (1): 4373, vii-viii.

18. Tamgadge S, Tamgadge A, Agre B. Writing oral pathology biopsy report: a guide for beginners. J Exp Ther Oncol. 2018; 12 (4): 273279.

19. Matiakis A, Karakostas P, Pavlou AM, Anagnostou E, Poulopoulos A. Angioleiomyoma of the oral cavity: a case report and brief review of the literature. J Korean Assoc Oral Maxillofac Surg. 2018; 44 (3): 136-139. doi: 10.5125/jkaoms.2018.44.3.136.

20. Eley KA, Alroyayamina S, Golding SJ, Tiam RN, Watt-Smith SR. Angioleiomyoma of the hard palate: report of a case and review of the literature and magnetic resonance imaging findings of this rare entity. Oral Surg Oral Med Oral Pathol Oral Radiol. 2012; 114 (2): e45-e49. doi: 10.1016/j.oooo.2012.01.014.

21. Arruda MM, Monteiro DY, Fernandes AM, Menegatti V, Thomazzi E, Hubner RA et al. Angioleiomyoma of the nasal cavity. Int Arch Otorhinolaryngol. 2014; 18 (4): 409-411. doi: 10.1055/s-00331364173.

22. Michael RC, Shah S. Angioleiomyoma of the nasal cavity. Indian J Microbiol Pathol. 2009; 52 (3): 386-388.
23. Barboza CAG, Ginani F, Leite-Segundo AV, Da Silva UH. Oral angioleiomyoma: a case report and considerations on differential diagnosisJ Bras Patol Med Lab. 2013; 49 (6): 429-432. doi: 10.1590/ s1676-24442013000600008.

24. Rawal SY, Rawal YB. Angioleiomyoma (vascular leiomyoma) of the oral cavity. Head Neck Pathol. 2018; 12 (1): 123-126. doi: 10.1007/ s12105-017-0827-9.

25. Osano H, loka Y, Okamoto R, Nakai Y, Hayashi H, Tsuchiya Y et al. Angioleiomyoma of the cheek: a case report. J Oral Sci. 2015; 57 (1): 63-66. doi: 10.2334/josnusd.57.63.

26. Morimoto N. Angiomyoma (vascular leiomyoma): a clinicopathologic study. Med J Kagoshima Univ. 1973; 24: 663-683.

Conflicto de intereses: Ninguno.

Aspectos éticos: Ninguno.

Financiamiento: Propio.

Correspondencia:

María Fernanda Partida González

E-mail: fernanda.partida@hotmail.com 